\title{
Enhancement in Optical Absorption of Plasmonic Solar Cells
}

\author{
Y. Premkumar Singh ${ }^{1,2}$, Anil Kumar ${ }^{1}$, Amit Jain ${ }^{3}$ and A. Kapoor ${ }^{1, *}$ \\ ${ }^{1}$ Department of Electronic Sciences, University of Delhi, South Campus, Benito Juarez Road, New Delhi-110021, India \\ ${ }^{2}$ Motilal Nehru College, University of Delhi, Benito Juarez Road, New Delhi-110021, India \\ ${ }^{3}$ Rajdhani College, University of Delhi, Raja Garden, New Delhi-110015, India
}

\begin{abstract}
Use of surface plasmons in photovoltaics is a recent and fast emerging field of interest of research that exploit the unique optical properties of metallic nano-structures. Using surface plasmons for guiding and localizing light at nanoscales can be used to improve optical absorption in thin-film solar cells. The present work focuses on the study of absorption enhancement using a periodic array of cylindrical silver nanowire placed on a thin silicon substrate. Studies have been made to optimize the particle size and the inter-particle distance for maximum absorption of AM1.5G solar radiation within the substrate. An enhancement with a factor of around 1.32 is observed for nanoparticles with a diameter of 140 $\mathrm{nm}$ and an inter-particle distance of $360 \mathrm{~nm}$. Blue-shifting of resonance wavelength with increasing inter-particle distance is observed. FDTD technique has been used for numerical modelling and investigation of plasmonic solar cells.
\end{abstract}

Keywords: Absorption enhancement, FDTD, plasmonic solar cell, surface plasmons.

\section{INTRODUCTION}

At present, thin-film solar cells which reduce both material and their processing cost significantly, have attracted the attention of optical researchers worldwide. Most of the thinfilm solar cells are based on silicon because of its stability, non-toxicity, well-developed technology and more abundance in nature. However, a major limitation with thin-film solar cells is the poor absorption of light as compared to wafer-based solar cells. It is well established that light-trapping by increasing its optical path length inside the absorbing film for obtaining high efficiency is crucial for thin-film solar cells. In light of the above restriction, a new method using plasmonic structures can improve the absorption of light due to excitation of localized surface plasmon [1-3]. Noble metals such as silver (Ag), gold (Au), aluminium (Al) and copper $(\mathrm{Cu})$, support surface plasmon due to their free electronlike behaviour [4]. Surface plasmon is the collective oscillations of excited free electrons of metallic particles [2, 5]. This unique property of metallic nano-structures can be used to enhance the optical absorption of solar cells through scattering of light and near-field concentration of light. The contribution of these mechanisms depends on the particle material, shape, size and refractive index of the surrounding dielectric [1-3]. These factors, together with the inter-particle distance, also determine optical resonances in coupled systems of plasmonic particles. Plasmon coupling takes place when the particles are closely spaced so that the electron oscillations in each particle are affected by the local field associated with the electron oscillations in the neighbouring

*Address correspondence to this author at the Department of Electronic Sciences, University of Delhi, South Campus, Benito Juarez Road, New Delhi-110021, India; Tel: +91-9350571397; Fax: +91-11-24119832;

E-mail: avinashi_kapoor@yahoo.com particles. This paper explores the effect of the particle size together with the inter-particle distance on the optical absorption due to plasmon coupling in a periodic array of $\mathrm{Ag}$ nanoparticle.

In a recent work, the dipole and quadrupole plasmon resonances have been qualitatively described for spherical and triangular Ag nanoparticles of different sizes [6]. Enhancement in red-shift and tunability of localized surface plasmon resonance has been demonstrated by introducing a coating medium to Ag island film [7]. The role of near-field coupling and Fano resonances in the scattering of light and transmission of light into $\mathrm{Si}$ substrate have been investigated using Ag nanoparticles [8]. An enhancement of 20-fold photocurrent was observed at $800 \mathrm{~nm}$ wavelength using silverisland particles on $165 \mathrm{~nm}$ thick SOI photodetectors [9]. A recent work reported an enhancement by a factor of 2.3 in external quantum efficiency of thin Si solar cells at $1100 \mathrm{~nm}$ wavelength using $\mathrm{Ag}$ nanoparticles [10]. An increase of $8.1 \%$ in short-circuit current density and $8.3 \%$ in energy conversion efficiency compared to values achieved in the a$\mathrm{Si}: \mathrm{H}$ layer without $\mathrm{Au}$ nanoparticles was observed [11]. A sevenfold enhancement of light absorption for wafer-based cells and up to 16-fold enhancement for $1.25 \mu \mathrm{m}$ thin SOI cells using Ag nanoparticles have been reported [12].

The present work focuses on the influence of particle size together with the inter-particle distance of a periodic array of cylindrical Ag nanowire on the optical absorption using FDTD method and compared with experimental works available in literature $[10-14,23]$. We reduce the $3-\mathrm{D}$ model to a 2-D one, since both models give similar results in simulations $[15,16]$. 2-D model of course reduce computational time and memory. It also allows us to investigate a larger parameter space in more detail. We have optimized the particle size and the inter-particles distance for maximum absorption of AM1.5G solar radiation within a $100 \mathrm{~nm}$ thick $\mathrm{Si}$ 


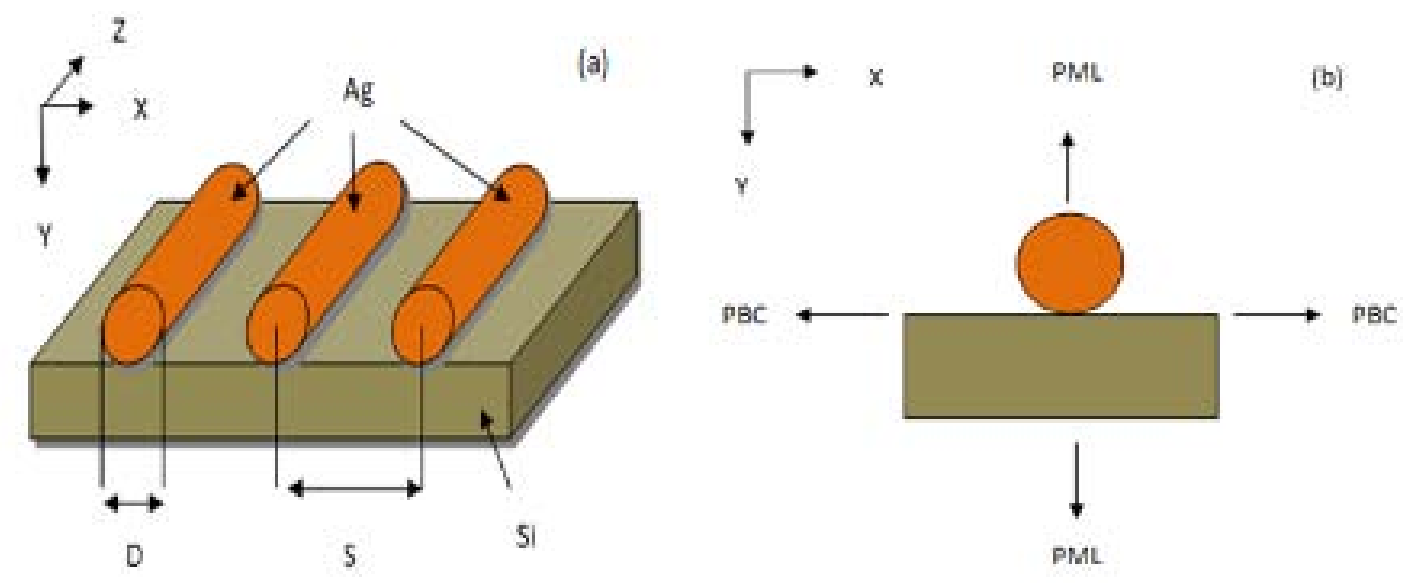

Fig. (1). (a) Basic structure of solar cell under investigation. (b) Schematic of the unit cell used for 2-D FDTD simulations.
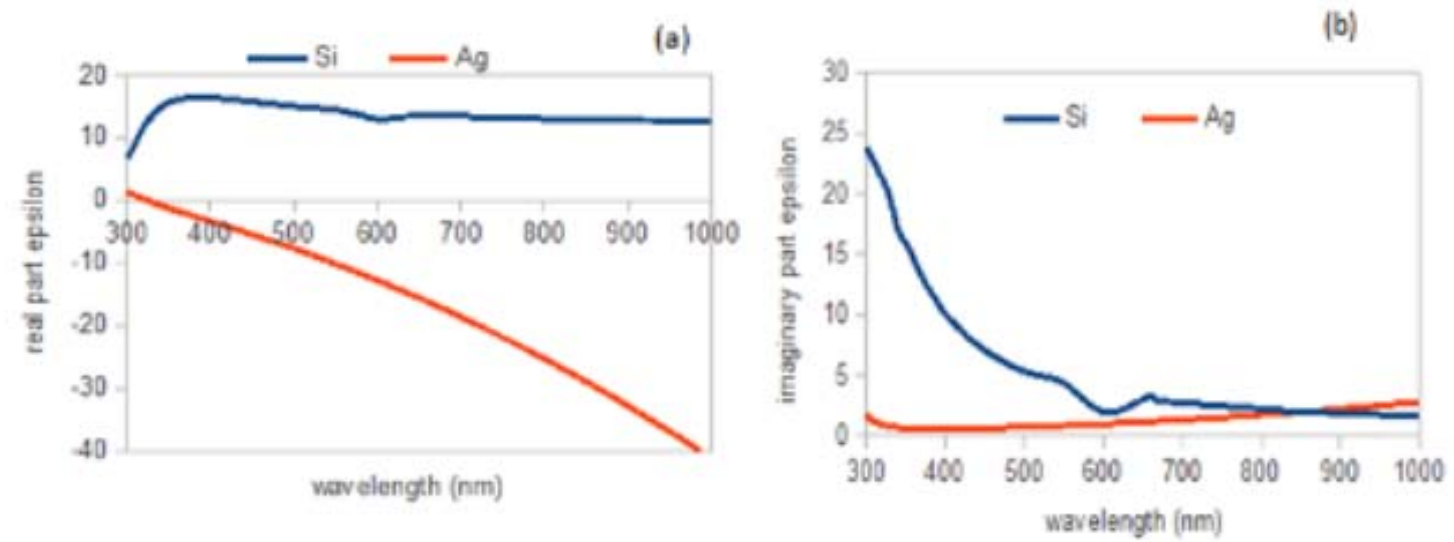

Fig. (2). Real part (a) and imaginary part (b) of dielectric constants of Si and Ag as function of wavelength.

substrate. Simulations were carried out for particle size ranging from $60-180 \mathrm{~nm}$ at an inter-particle distance ranging from $70-500 \mathrm{~nm}$.

\section{MATERIALS AND METHODOLOGY}

Numerical simulations were performed with 2-D finitedifference time-domain (FDTD) method using MEEP. FDTD [17] is a widely used technique for computational electromagnetism in which space and time are divided into a regular discrete grid and simulates the time evolved fields. In 1966, Yee [18] introduced the basis of FDTD numerical technique, which exploits the mutual dependence of the $\mathrm{E}$ and $\mathrm{H}$ fields in time and space over a discrete spatial grid for solving Maxwell's curl equations. Within the last two decades, FDTD gain rapidly increasing interest for the simulation of complex and largely inhomogeneous structures due to its systematic and explicit approach. It can be used to investigate a large variety of electromagnetic wave interaction problems accurately. Since it is a time domain method, FDTD solutions can cover a wide frequency range with a single simulation run. However, FDTD simulations take very long solution times, since the grid spatial discretization of the entire computational domain must be sufficiently fine to resolve both the smallest electromagnetic wavelength and the smallest geometrical feature in the model.

MEEP (MIT Electromagnetic Equation Propagation) [19] is a free, open-source simulation software package for FDTD method. Its features include simulation in Cartesian $(1 \mathrm{~d} / 2 \mathrm{~d} / 3 \mathrm{~d})$ and cylindrical co-ordinates, dispersive electric and magnetic media, arbitrary material and source distributions, a variety of boundary conditions (PML, Bloch-periodic and perfect conductor), exploitation of symmetries to reduce the computation size and time. It also includes field analyses (flux spectra, local density of states and energy integrals) and field output in the HDF5 standard scientific data format, supported by many visualization tools.

Fig. (1a). shows the basic structure of solar cell under investigation which consists of a periodic array of cylindrical Ag nanowire on top of the Si substrate. The 2-D unit cell used for simulation study is shown schematically in Fig. (1b). It consists of a single cylindrical Ag nanowire on a $100 \mathrm{~nm}$ thick layer of Si. Perfectly matched layers (PMLs) were used in Y-direction boundaries to prevent interference effect, and periodical boundary condition (PBC) was used in $\mathrm{X}$-direction to model periodic array of nanowire. A grid size of $1 \mathrm{~nm}$ was used in the entire computational domain. Even mirror symmetry along X-direction was used to reduce the computation time. A plane wave of polarization along nanowire array $(\mathrm{X})$ axis was incident normally on the cell surface. We used the wavelength-dependent dielectric constants of $\mathrm{Si}$ and $\mathrm{Ag}$ modelled [20,21] respectively using Drude-Lorentz equation that are plotted in Fig. (2). real part (a) and imaginary part (b). 


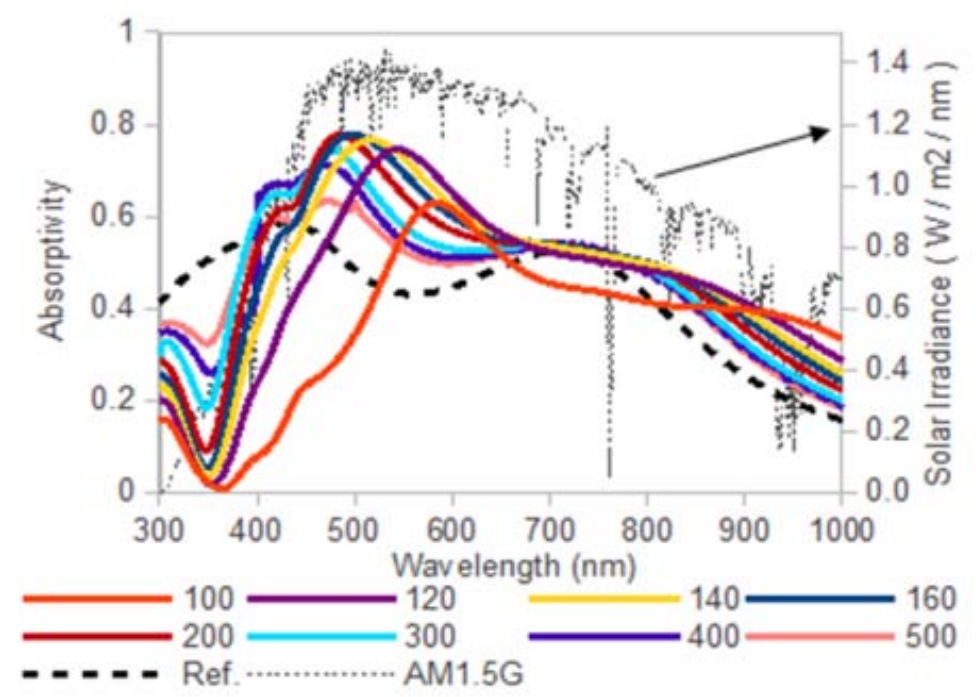

Fig. (3). Absorption spectra in the Si substrate with cylindrical $\mathrm{Ag}$ nanowires of $\mathrm{D}=80 \mathrm{~nm}$ at various inter-particle distance, $\mathrm{S}$ (in $\mathrm{nm}$ ).

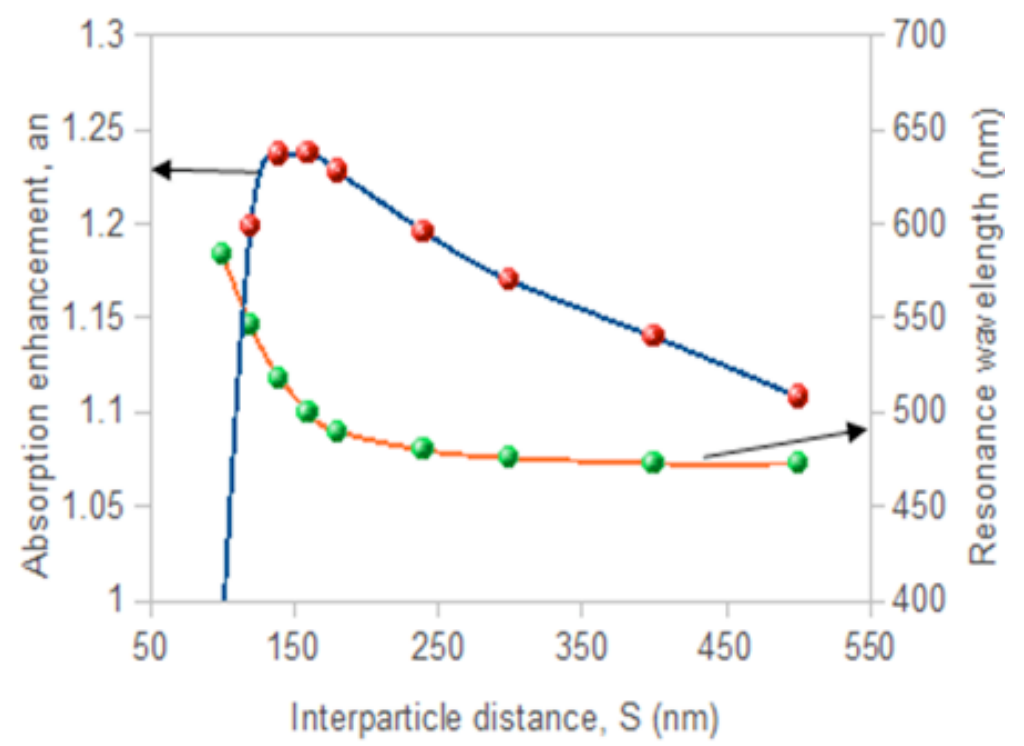

Fig. (4). Variation of the absorption enhancement ratio, $a_{n}$ and the resonance wavelength, $\lambda_{0}$ with inter-particle distance, $S$ for Ag nanoparticles of $\mathrm{D}=80 \mathrm{~nm}$.

By monitoring the electromagnetic flux on top, $e_{I}(\lambda)$ and bottom, $e_{2}(\lambda)$ surface planes of the substrate, the fraction of light absorbed (absorptivity) within the $\mathrm{Si}$ substrate was evaluated by $\left[e_{1}(\lambda)-e_{2}(\lambda)\right] / e_{i n}(\lambda)$, where $e_{i n}(\lambda)$ is the incident flux $[22,23]$. Flux was determined by integrating the poynting vector of the fourier-transformed fields over the plane for each frequency. Absorption enhancement ratio, $a_{n}$ within the spectral range of $300-1000 \mathrm{~nm}$ was calculated as

$a_{n}=\frac{\int_{300}^{1000} \alpha_{n}(\lambda) S(\lambda) d \lambda}{\int_{300}^{1000} \alpha_{0}(\lambda) S(\lambda) d \lambda}$

where $S(\lambda)$ is the irradiance spectrum of AM1.5G, $\alpha_{n}(\lambda)$ and $\alpha_{0}(\lambda)$ are the absorptivity within the Si substrate with and without nanoparticles respectively.

\section{RESULTS AND DISCUSSION}

Fig. (3) shows the absorption spectra in the Si substrate with a periodic array of cylindrical Ag nanowire of diameter,
$\mathrm{D}=80 \mathrm{~nm}$ at various inter-particle distances, $\mathrm{S}$. Note that this distance is measured from the centre of the particles. Absorption spectrum without nanoparticles as reference and AM1.5G solar spectrum are also included. The strength of coupling between surface plasmons of metallic nanoparticles depends on the inter-particle separation. For closely spaced particles, a strong coupling causes a broadening of plasmon resonance and a large nonradiative loss, resulting in a weak absorption [16]. Moreover, for widely spaced particles, the effect of nanoparticles can be almost disregarded and the structure converges to the case with no nanoparticles. A high absorption region appears at optimized spacing indicating the existence of an optimal inter-particle distance for maximum absorption. This phenomenon is depicted in Fig. (4). The absorption enhancement ratio increases sharply to its peak and decay slowly with inter-particle distance. In the present case, the optimum inter-particle distance comes out to be $\sim 160 \mathrm{~nm}$. We can also observe a remarkable blue-shift of the resonance wavelength, $\lambda_{0}$ as the inter-particle distance increases (Fig. 4). This behaviour is already reported [24] and 


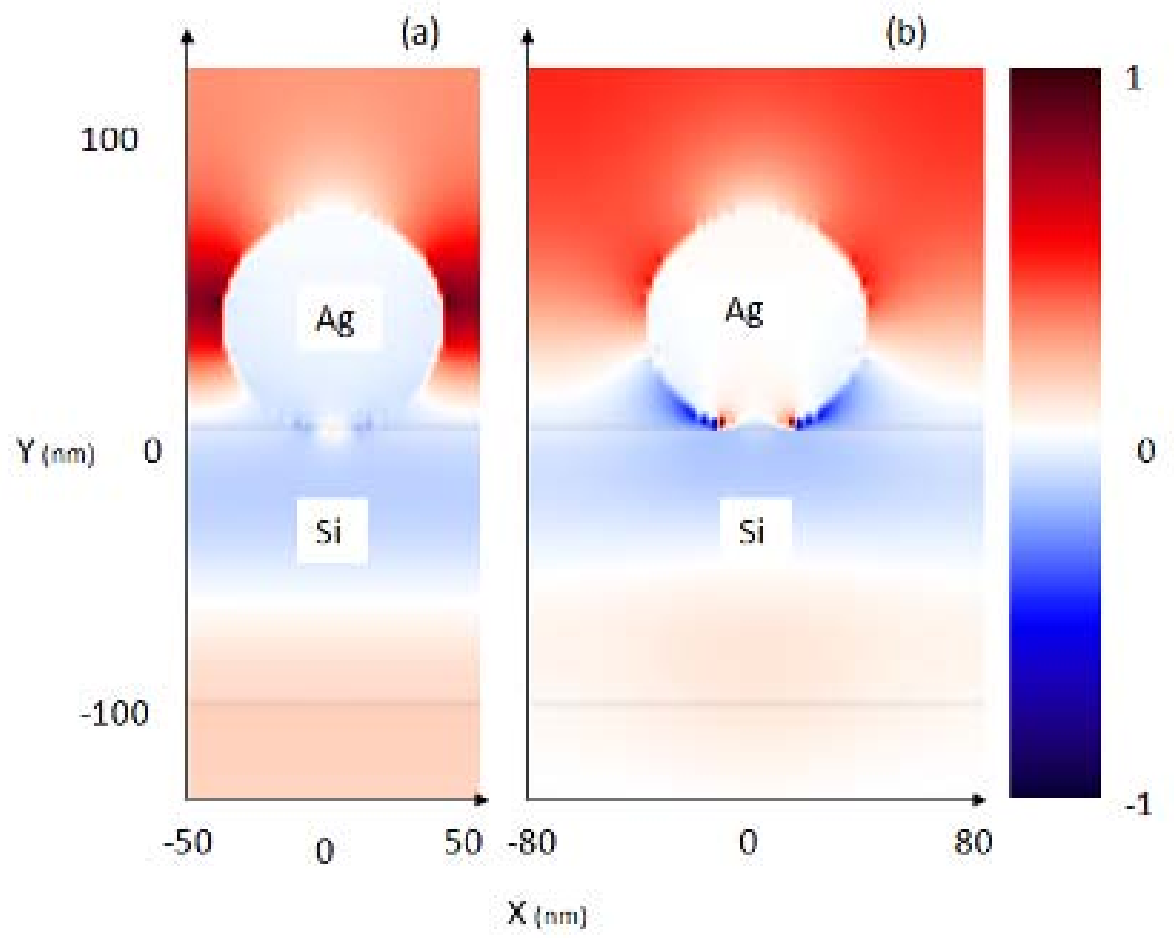

Fig. (5). Spatial distribution of $\mathrm{x}$-component of electric field with a periodic Ag nanoparticles of $\mathrm{D}=80 \mathrm{~nm}$ on the $\mathrm{Si}$ substrate (a) $S=100$ $\mathrm{nm}, \lambda_{0}=584 \mathrm{~nm}$ and (b) $S=160 \mathrm{~nm}, \lambda_{0}=500 \mathrm{~nm}$.

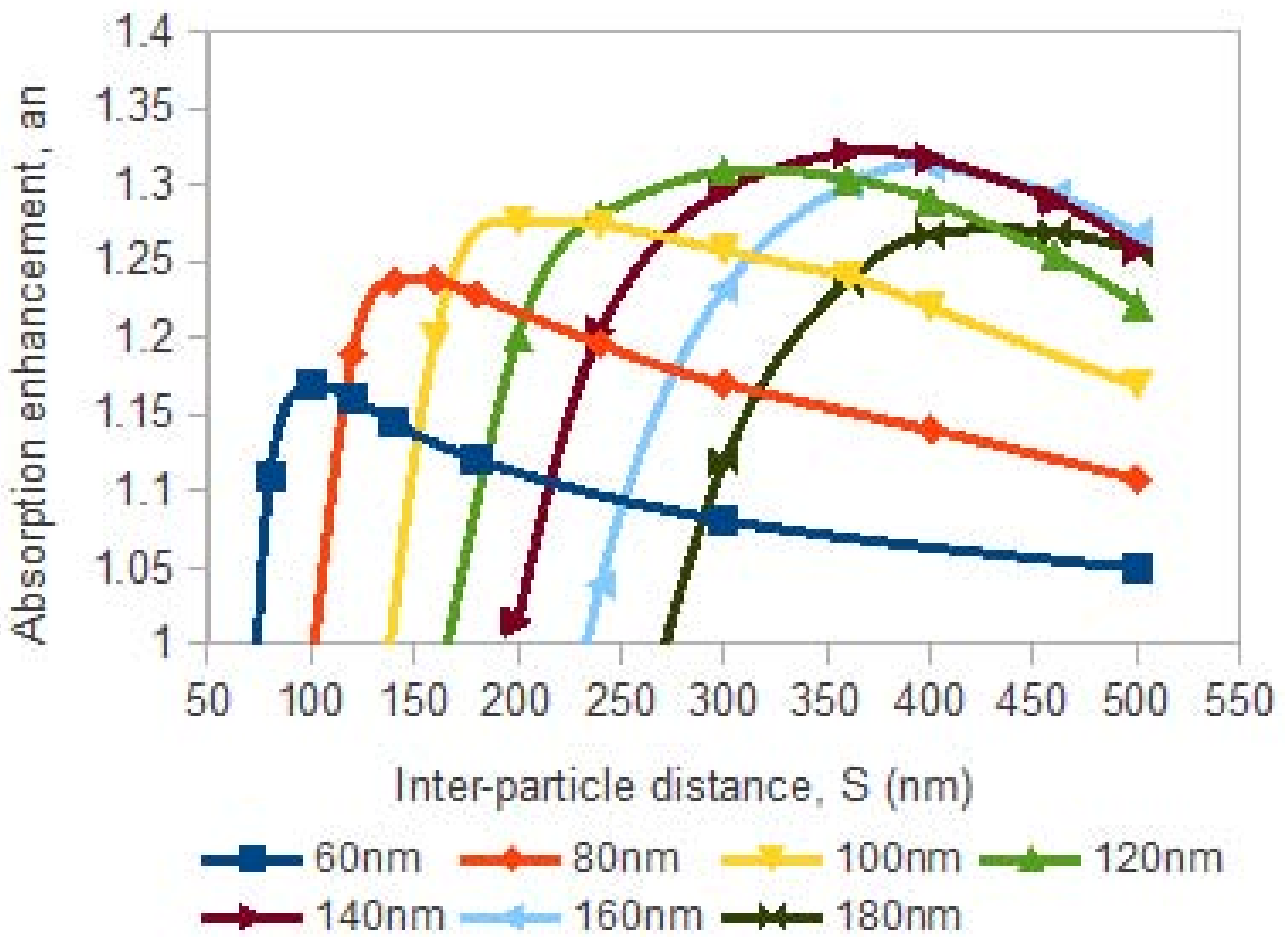

Fig. (6). Value of the absorption enhancement ratio for nanosparticles of various diameters as a function of inter-particle distance.

has been explained by a simple dipole-dipole interaction model. Fig. (5) shows the spatial distribution of $\mathrm{x}$-component of electric field for (a) $\mathrm{S}=100 \mathrm{~nm}, \lambda_{0}=584 \mathrm{~nm}$ and (b) $\mathrm{S}=160 \mathrm{~nm}, \lambda_{0}=500 \mathrm{~nm}$. The field distributions are clearly dominated by the excitation of a dipolelike resonance in the nanoparticle. For $\mathrm{S}=100 \mathrm{~nm}$, concentration of high density field in the gap between the nanoparticles is observed. This high density field is spread out in the case of $\mathrm{S}=160 \mathrm{~nm}$.
Fig. (6) shows the value of absorption enhancement ratio for nanoparticles of various diameters as a function of $\mathrm{S}$. For smaller particles, light absorbed by the particle is dominated over scattering, so the absorption enhancement ratio is relatively low. Scattering starts to dominate as the particle size increases. In this case, the optimal size for maximum absorption within the substrate comes out to be $\sim 140 \mathrm{~nm}$. The conduction electrons across too large particle do not move in 


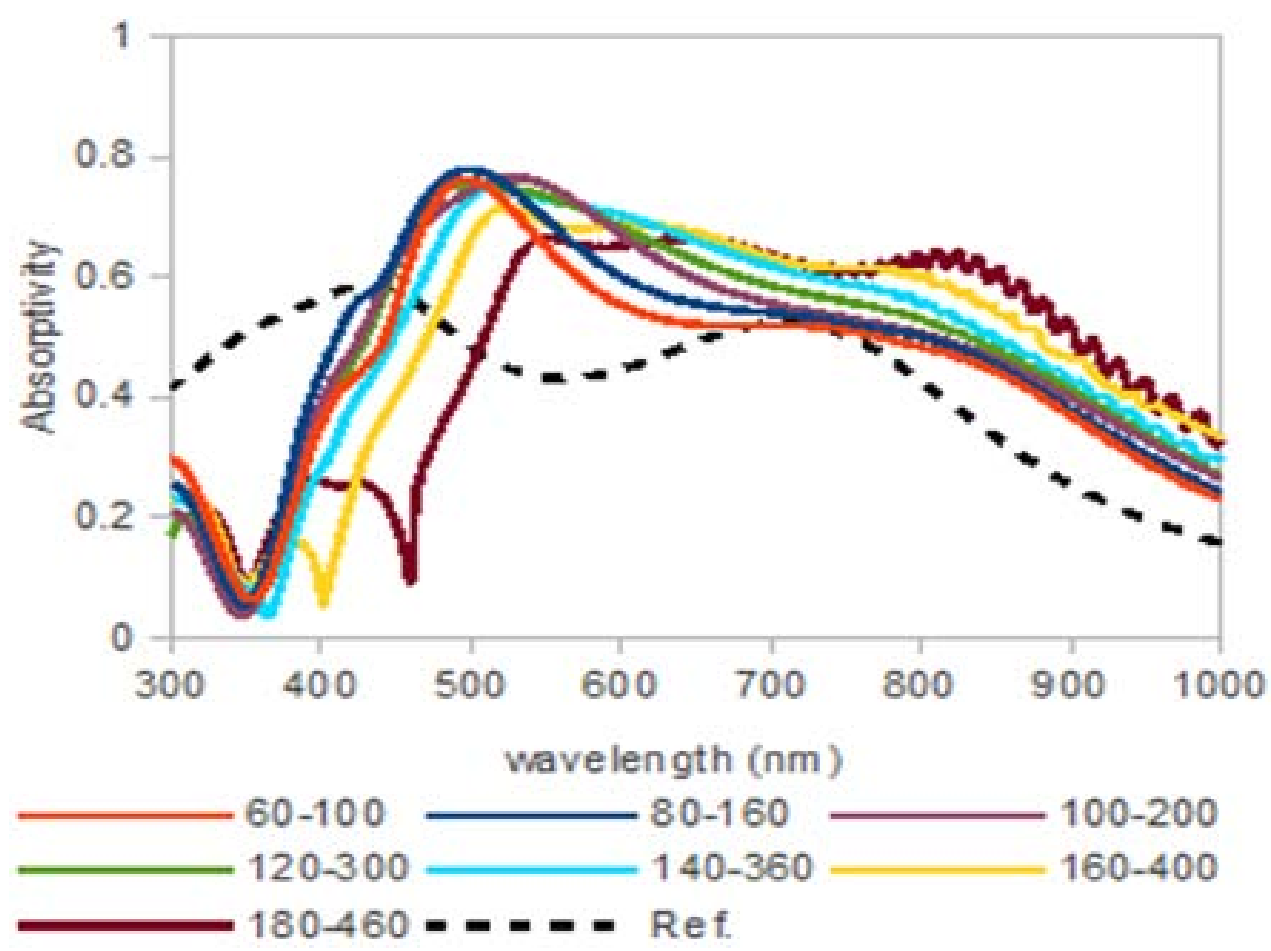

Fig. (7). Absorption spectra for nanoparticles of various diameters at their optimal spacing. In the legend, the first number indicates the diameter of particles (in $\mathrm{nm}$ ) and second indicates the inter-particle distance (in $\mathrm{nm}$ ).

phase which leads to a reduction in the depolarization of field at the centre of the particle, thereby reducing scattering [3]. It can also be observed that the optimum spacing between the particle surfaces increases as the particle become larger. Absorption spectra for nanoparticles of various diameters at their optimal spacing are shown in Fig. (7). Broad plasmon resonance and excitation of higher order plasmon modes (quadrupole, octupole) can be clearly observed for large size particles due to dynamic depolarization and radiative damping effects [3].

\section{CONCLUSION}

Enhancement in optical absorption of AM1.5G solar radiation within a thin Si substrate have been analyzed using a periodic array of cylindrical $\mathrm{Ag}$ nanowire, keeping in mind the application of surface plasmons in thin-film solar cells. It has been shown that the size of the nanoparticles should be sufficiently large so that scattering of light dominates over light absorbed by the particles. Moreover, the inter-particle distance also determines optical absorption in coupled systems of nanoparticles. The inter-particle distance of a periodic array of nanoparticles has been optimized to yield maximum absorption within the substrate for cylindrical nanowires of diameter ranging from $60 \mathrm{~nm}$ to $180 \mathrm{~nm}$. The best enhancement with a factor of around 1.32 is observed for nanoparticles with a diameter of $140 \mathrm{~nm}$ and an interparticle distance of $360 \mathrm{~nm}$, bringing the structure to a much thicker cell without nanoparticles.

\section{CONFLICT OF INTEREST}

The authors confirm that this article content has no conflicts of interest.

\section{ACKNOWLEDGEMENT}

Declared none.

\section{REFERENCES}

[1] Atwater, H. A.; Polman, A. Plasmonics for improved photovoltaic devices. Nat. Mater., 2010, 9, 205-213.

[2] Pillai, S.; Green, M. A. Plasmonics for photovoltaic applications, Solar. Energy Mater. Solar. Cells, 2010, 94, 1481-1486.

[3] Catchpole, K. R.; Polman, A. Plasmonic solar cells. Opt. Express, 2008, 16 (26), 21793-21800.

[4] Maier, S. A.; Brongersma, M. L.; Kik, P. G.; Requicha, A. A.; Atwater, H. A. Plasmonics- A route to nanoscale optical devices. Adv. Mater., 2001, 13 (19), 1501-1505.

[5] Murray, W. A.; Barnes, W. L. plasmonic materials. Adv. Mater. 2007, 19, 3771-3782.

[6] Kelly, K. L.; Coronado, E.; Zhao, L. L.; Schatz, G. C. The optical properties of metal nanoparticles: the influence of size, shape, and dielectric environment. J. Phys. Chem. B, 2003, 107, 668-677.

[7] Hu, G.; Tazawa, M.; Jin, P.; Nakao, S.; Yashimura, K. Wavelength tuning of surface Plasmon resonance using dielectric layers on silver island films. Appl. Phys. Lett., 2003, 82 (22), 3811-3813.

[8] Spinelli, P.; Lare, C. V.; Verhagen, E.; Polman, A. Controlling Fano lineshapes in plasmon-mediated light coupling into a substrate. Opt. Express, 2011, 19 (S3), A303-A311.

[9] Stuart, H. R.; Hall, D. G. Island size effects in nanoparticleenhanced photodetectors. Appl. Phys. Lett., 1998, 73 (26), 38153817.

[10] Beck, F. J.; Polman, A.; Catchpole, K. R. Tunable light trapping for solar cells using localized surface plasmons. J. Appl. Phys., 2009, 105, 114310-1-114310-7.

[11] Derkacs, D.; Lim, S. H.; Matheu, P.; Mar, W.; Yu, E. T. Improved performance of amorphous silicon solar cells via scattering from surface Plasmon polaritons in nearby metallic nanoparticles. Appl. Phys. Lett., 2006, 89, 093103.1-093103.3.

[12] Pillai, S.; Catchpole, K. R.; Trupke, T.; Green, M. A. Surface Plasmon enhanced silicon solar cells. J. Appl. Phys., 2007, 101, 093105-1-093105-8.

[13] Schaadt, D. M.; Feng, B.; Yub, E. T. Enhanced semiconductor optical absorption via surface plasmon excitation in metal nanoparticles. Appl. Phys. Lett., 2005, 86, 063106-1-063106-3.

[14] Lamprecht, B; Schider, G.; Lechner, R. T.; Ditlbacher, H.; Krenn, J. R.; Leitner, A.; Aussenegg, F. R. Metal nanoparticle gratings: influence of dipolar particle interaction on the plasmon resonance. Phys. Rev. Lett., 2000, 84 (20), 4721-4724. 
[15] Moreno, F.; Camara, B. G.; Saiz, J. M.; Gonzalez, F. Interaction of nanoparticles with substrates: effects on the dipolar behaviour of the particles. Opt. Express. 2006, 16 (17), 12487-12504.

[16] Rockstuhl, C. R.; Fahr, S.; Lederer, F. Absorption enhancement in solar cells by localized Plasmon polaritons. J. Appl. Phys., 2008, 104, 123102-1-123102-7.

[17] Taflove, A.; Hagness, S. C. Computational Electrodynamics: The Finite-Difference Time Domain Method. $2^{\text {nd }}$ ed.; Artech House: Norwood, M A, 2000

[18] Yee, K. S. Numerical solution of initial boundary value problems involving maxwell's equations in isotropic media. IEEE Trans. Antennas Propag., 1966, AP-14(3), 302-307.

[19] Oskooi, A. F.; Roundy, D.; Ibanescu, M.; Bermel, P.; Joannopoulos, J. D.; Johnson, S. G. Meep: A flexible free-software package for electromagnetic simulations by the FDTD method. Comput. Phys. Commun., 2010, 181, 687-702.
[20] Pirozhenko, I.; Lambrecht, A. Influence of slab thickness on the Casimir force. Phys. Rev. A, 2008, 77, 013811-1-013811-8.

[21] Rakic, A. D.; Djurisic, A. B.; Elazar, J. M.; Majewski, M. L. optical properties of metallic films for vertical-cavity optoelectronic devices. Appl. Opt., 1998, 37 (22), 5271-5283.

[22] Duche, D.; Torchio, P.; Escoubas, L.; Monestier, F.; Simon, J. J.; Flory, F.; Mathian, G. Improving light absorption in organic solar cells by plasmonic contribution. Solar. Energy Mater. Solar. Cells, 2009, 93, 1377-1382.

[23] Zhao, D.; Ma, Z.; Zhou, W. Plasmonic field and efficiency enhancement in crystalline thin film photovoltaics. Proc. SPIE, 2010, 7772, 777215-1-777215-8.

[24] Rechberger, W.; Hohenau, A.; Leitner, A.; Krenn, J. R.; Lamprecht, B.; Aussenegg, F. R. Optical properties of two interacting gold nanoparticles. Opt. Commun., 2003, 220, 137-141.

Received: December 23, 2012

Revised: January 11, 2013

Accepted: January 12, 2013

(C) Singh et al.; Licensee Bentham Open.

This is an open access article licensed under the terms of the Creative Commons Attribution Non-Commercial License (http://creativecommons.org/licenses/by-nc/3.0/) which permits unrestricted, non-commercial use, distribution and reproduction in any medium, provided the work is properly cited. 